\title{
Quantum field theory treatment of magnetic effects on a system of free electrons
}

\author{
C. Verzegnass: ${ }^{*}$ \\ Politecnico di Ingegneria e Architettura, University of Udine, Udine, Italy, \\ and $A M e C$ (Association for Medicine and Complexity), Trieste, Italy \\ R. Germano \\ PROMETE CNR Spin Off, Napoli, Italy \\ P. Kurian \\ National Human Genome Center and Department of Medicine, \\ Howard University College of Medicine, Washington DC, \\ USA; Computational Physics Laboratory, Howard University, Washington DC, USA
}

\begin{abstract}
The effects of a magnetic field on the energy and on the spin of free electrons are computed in the framework of quantum field theory. In the case of a constant moderate field and with relatively slow electrons, the derived formulae are particularly simple. A comparison with the approaches of classical physics and of quantum mechanics shows essential differences and important analogies. The relevance to the magnetic effects of the initial polarization components of the electron states and the possible existence of special values of these quantities are discussed in the final conclusions, which might be useful to explain recent experiments on quasi-free electrons in chiral systems in biology.
\end{abstract}

Two of us have described in a recent letter [1] the reciprocal and compensatory conversion between the spin and orbital angular momentum of a free electron induced by a magnetic field in the theoretical framework of quantum electrodynamics. To describe a more realistic system, with a crowd of electrons and atomic nuclei in full generality, the elementary properties of these constituents should determine the results of the process of interaction with the magnetic field, even if emergent or novel effects arise at each scale of organization in the system. However, the role of the mutual interactions between electrons and nuclei will make the calculation of the purely magnetic effects hardly performable. An almost obvious statement is that the suggested calculation might be more easily carried out if the mutual interaction between these constituents were extremely weak, such that the interaction might be ignored altogether. The simplest scenario would be the case of a system of free electrons, of which there are abundant examples in solid-state, condensed matter, and biological physics.

In this spirit we have considered the goal of computing the effects of a magnetic field on a system of completely free electrons, considering this calculation as an initial step to which suitably weak interactions can be added later. To summarize these effects, we shall now review the main expressions for a single electron state that are required to obtain the principal results for a system of free electrons. In our work, we have followed the quantum field theory notations and conventions of Peskin and Schroeder [2].

For a single electron state we shall choose as basic quantities the four complex components of the electron field $\psi(\mathbf{x})$, written as $\psi_{s}(t, \vec{x})$, where $s=1,2,3,4$. Using the explicitly hermitian form of the Dirac Lagrangian, we have rewritten below the total energy in terms of these components:

$$
\begin{aligned}
\mathcal{H}=\int d^{3} x & {\left[-\frac{i}{2} \bar{\psi} \gamma^{k} \partial_{k}+\frac{i}{2}\left(\partial_{k} \bar{\psi}\right) \gamma^{k}+m_{e} \bar{\psi}\right] \psi } \\
=\int d^{3} x \quad & {\left[2 m_{e} \operatorname{Re}\left(\psi_{1}^{*} \psi_{3}+\psi_{2}^{*} \psi_{4}\right)-\operatorname{Im}\left(\psi_{1}^{*} \partial_{3} \psi_{1}+\psi_{1}^{*}\left(\partial_{1}-i \partial_{2}\right) \psi_{2}+\psi_{2}^{*}\left(\partial_{1}+i \partial_{2}\right) \psi_{1}-\psi_{2}^{*} \partial_{3} \psi_{2}\right)+\right.} \\
& \left.\operatorname{Im}\left(\psi_{3}^{*} \partial_{3} \psi_{3}+\psi_{3}^{*}\left(\partial_{1}-i \partial_{2}\right) \psi_{4}+\psi_{4}^{*}\left(\partial_{1}+i \partial_{2}\right) \psi_{3}-\psi_{4}^{*} \partial_{3} \psi_{4}\right)\right] .
\end{aligned}
$$

Consistent with the defined quantities and notations for the changes in the electron field components with the introduction of purely magnetic potentials $\vec{A}$, we also derived the effects of these potentials on spin [1] and energy [3]. In

*Electronic address: claudio@ts.infn.it

†Electronic address: pkurian@howard.edu 
the non-relativistic limit (NRL) where we are concerned $\left(\psi_{1}, \psi_{2} \gg \psi_{3}, \psi_{4}\right)$,

$$
\Delta_{\vec{A}} \mathcal{H} \stackrel{\mathrm{NRL}}{\longrightarrow}-4|e| \int d^{3} x \vec{A} \cdot \vec{s}^{\prime}
$$

where $e$ is the electron charge and $\vec{s}^{\prime}$ is the "spin current" in the NRL. The spin current is defined generally as the quantity whose integral gives the spin vector itself, $\vec{S}=\int d^{3} x \vec{s}$, and its components are

$$
\begin{aligned}
& s_{1}=\operatorname{Re}\left(\psi_{1}^{*} \psi_{2}+\psi_{3}^{*} \psi_{4}\right) \\
& s_{2}=\operatorname{Im}\left(\psi_{1}^{*} \psi_{2}+\psi_{3}^{*} \psi_{4}\right) \\
& s_{3}=\frac{1}{2}\left(\psi_{1}^{*} \psi_{1}-\psi_{2}^{*} \psi_{2}+\psi_{3}^{*} \psi_{3}-\psi_{4}^{*} \psi_{4}\right) .
\end{aligned}
$$

A short discussion of Equation (2) will be relevant here. Consider the expression that would have been predicted for the magnetic effect on the energy by a strictly quantum mechanical approach. As is well known, this expressioncalled the Zeeman effect - can be written for an electron as a scalar product between the electron angular momentum $\vec{J}$ and the magnetic field $\vec{B}$. For the contribution coming from the electron spin $\vec{S}$, we would have

$$
\Delta_{\vec{B}} \mathcal{H}=-g_{s} \mu_{B} \vec{B} \cdot \vec{S}
$$

where $g_{s} \approx 2$ is the gyromagnetic factor and $\mu_{B}=|e| / 2 m_{e}$ is the Bohr magneton. One can see a faint resemblance between Equation (4) above and the quantum field theory expression of Equation (2), with (roughly speaking) a replacement of the magnetic field $\vec{B}$ with the magnetic potentials $\vec{A}$ and likewise of the spin $\vec{S}$ with the spin current $\vec{s}^{\prime}$. Using the same quantum field theory framework, we have previously derived the effect of a magnetic field on the electron spin, which in full generality is given by the following expression:

$$
\Delta_{\vec{A}} \vec{S}=|e| \int d \vec{x}\left[\vec{A} \times \vec{\rho}_{E}\right],
$$

where $\vec{\rho}_{E}$ is defined as

$$
\vec{\rho}_{E}=\frac{i}{m_{e}} \psi^{\dagger} \vec{\gamma} \psi
$$

with $\vec{\gamma}$ including the conventional gamma matrices. Such an expression in Equation (5) is only possible in quantum field theory, where the value for the spin in terms of the fields is known (see Equations (33)).

We then computed the expectation value of the change in spin for a single electron state. More precisely, we have considered a properly normalized free electron state of momentum $\vec{k}=\left(0,0, k_{3}\right)$ along the $z$ axis, in a linear superposition of spin eigenstates:

$$
|\Psi(\vec{k})\rangle=\lambda_{+}|\uparrow, \vec{k}\rangle+\lambda_{-}|\downarrow, \vec{k}\rangle .
$$

Under the assumption that the components of the magnetic potential remain reasonably close to their average values in the finite volume of dimension $d$ where the applied magnetic potential is non-vanishing, we obtained the following result for the expectation value of the change for the third component of spin:

$$
\left\langle\Delta_{\vec{A}} S_{3}\right\rangle=\frac{|e|}{m_{e}} B_{3} d\left[\operatorname{Re}\left(\lambda_{+} \lambda_{-}^{*}\right)+\operatorname{Im}\left(\lambda_{+} \lambda_{-}^{*}\right)\right],
$$

where $\lambda_{+}, \lambda_{-}$are the complex coefficients defining a linear superposition of spin eigenstates.

There are two features of Equation (8) that should be highlighted. First, in classical physics there exists a known effect that a static magnetic field $B$ has on the motion of a magnetic moment. This motion is characterized by the well-known Larmor precession, where the magnetic moment rotates about the magnetic field vector, describing a cone around the axis of the applied field. In the rotation, the Larmor angular velocity $\omega_{L}$ has the value

$$
\omega_{L}=g_{s} \mu_{B} B
$$

Without loss of generality, the magnetic field can be oriented along the $z$ axis and therefore in our discussion $B$ can be equated with $B_{3}$. The circular motion of the magnetic moment on a circumference of radius $r$ perpendicular to the field direction has a velocity $v$ given by the usual expression for centripetal motion:

$$
v=r \omega_{L} .
$$


During the motion, the magnetic moment acquires an orbital angular momentum whose component $L_{z}$ in the direction of the field is given by the classical formula

$$
L_{z}=M v r=\left(M r^{2}\right) \omega_{L} .
$$

The acquired angular momentum component $L_{z}$ is therefore proportional to the Larmor frequency $\omega_{L}$.

If the considered system is a particle with spin angular momentum, it would be reasonable to suppose that a similar effect might appear for its spin component along the field, since its interaction with the field is analogous to that of a magnetic moment. In fact, this effect in the quantum field theory description is given by Equation (8), which can be rewritten as

$$
\left\langle\Delta_{\vec{A}} S_{3}\right\rangle=\left(g_{s} \mu_{B} B_{3}\right) d\left[\operatorname{Re}\left(\lambda_{+} \lambda_{-}^{*}\right)+\operatorname{Im}\left(\lambda_{+} \lambda_{-}^{*}\right)\right]=\omega_{L} d f\left(\lambda_{+}, \lambda_{-}\right) .
$$

As possibly expected, the resulting spin effect in Equation (12) is actually proportional to $\omega_{L}$, which is certainly analogous to the classical result for a magnetic moment described in Equation (11). Note well that in the equation above we have defined the special function $f\left(\lambda_{+}, \lambda_{-}\right)$, which will become relevant in the discussion that follows.

The second feature of Equation (8) concerns the identification of the quantities that produce the magnetic effect. One of these is, as one would expect, the magnetic field strength $B_{3}$. For the electron contribution, besides the charge-to-mass ratio, one can see that it is produced by a special function $f$ of the electron spin-up and spin-down polarization components, $\lambda_{+}$and $\lambda_{-}$. Given the fact that $f$ determines the observable effect, it seems natural to us to associate to this factor a specific name that is descriptive of the phenomenon. One can express these features by saying that $f$ behaves like a "chirality index" (CI). In this spirit, we shall identify it with this CI notation and say that the average electron $S_{z}$ magnetic variation is proportional to the electron CI.

As a first simplified case, we consider a situation in which both components $\lambda_{+}$and $\lambda_{-}$are real. The surviving effect in Equation (8) is then proportional to the product $\lambda_{+} \lambda_{-}$. This simplified CI has the following special features: (A) It vanishes when the electron state is fully polarized (maximum chirality), either in the extreme right-handed state $\left(\lambda_{+}=1\right)$ or in the extreme left-handed state $\left(\lambda_{-}=1\right)$. (B) It has a maximum $(1 / 2)$ in the most symmetrical situations where $\lambda_{+}$and $\lambda_{-}$are equal (no chirality), with modulus $1 / \sqrt{2}$, as imposed by the completeness relation for the superposed spin state. (C) It has a minimum $(-1 / 2)$ in the most anti-symmetrical situations where $\lambda_{+}$and $\lambda_{-}$ are opposite (also no chirality), again with modulus $1 / \sqrt{2}$. The features $(\mathrm{A}),(\mathrm{B})$, and $(\mathrm{C})$ thus make the connection between the CI and the values of the probability amplitudes for the electron spin state.

A related question that arises at this point is the determination of the average magnetic variation of the electron energy $\mathcal{H}$. Assuming identical properties of the magnetic field previously considered and taking the same kind of electron state, with $E_{0} \gg m_{e}$ (but still in the NRL), one finds for the average energy magnetic variation the following expression:

$$
\left\langle\Delta_{\vec{A}} \mathcal{H}\right\rangle=\frac{2 m_{e}|e|}{E_{0}} B_{3} d\left[\operatorname{Re}\left(\lambda_{+} \lambda_{-}^{*}\right)+\operatorname{Im}\left(\lambda_{+} \lambda_{-}^{*}\right)\right]
$$

where, again, $d$ is the dimension of the volume where the magnetic field and the electron interact non-negligibly. The main conclusion to be gleaned from Equation (13) is that the electron CI also determines the result for the average magnetic variation of the electron energy, as it did with the spin angular momentum. We can therefore conclude that the electron CI is an essential electron quantity in the quantum field theory determination of the magnetic effects on a free electron state.

It remains an open question whether for a free electron state (or a system of free electrons) the value of its CI may be arbitrary or rather fixed by a general rule. Equation (13) gives the energy that the electron will absorb from the applied magnetic field, and given the fact that the electron is almost free, it appears to us reasonably motivated that its absorption from the magnetic field should be maximal. If this assumption is accepted, the immediate consequence is that $\left|\operatorname{Re}\left(\lambda_{+} \lambda_{-}^{*}\right)+\operatorname{Im}\left(\lambda_{+} \lambda_{-}^{*}\right)\right|$ attains its maximum value of $1 / 2$, or in the simplified case of real coefficients,

$$
\lambda_{+}=\lambda_{-}= \pm 1 / \sqrt{2}
$$

Equation (14) is valid for a single electron state. In our opinion, if one considers a system of completely free electrons that interact with a magnetic field, the requirement of maximum energy absorption should continue to be valid for each separate electron, since it does not interact even weakly with the other components of the system. Thus for a system of free electrons, each CI of the various electron components should retain the value of Equation (14). Alternatively stated, for each electron of the system, the probability that its spin component is found in the direction of the field will be equal to the probability that it is found in the opposite direction. This conclusion is indeed satisfying since it is in total agreement with the results of the historical Stern-Gerlach experiment. 
In a strictly quantum mechanical description of a system of free electrons, the system acquires from its interaction with a magnetic field $B$ a quantity called magnetic susceptibility due to the fact that the number of electrons with spin in the direction of the field is different from the number of electrons with spin in the opposite direction. The spin probability amplitude is not changed by the introduction of a magnetic field. On the contrary, one can conclude from Equation (8) that the average value of the increase of the electron spin component in the direction of the magnetic field is proportional to the magnetic field component, and this value can be larger or smaller than the spin component in the opposite direction, depending on the values of the spin probabilities that determine the state polarization. This represents a quantum field theory generalization of the quantum mechanics result.

Clearly, in a biological situation the states to be considered are much more complicated than free electron systems with specific CIs. However, the role of spin in biology may still be ubiquitous, as recent studies have confirmed the spin selectivity of electron transport through chiral molecules [4, 5] such as DNA and proteins. Furthermore, recent experimental findings by one of us obtained in V. Elia's research group [6] show that supramolecular structures can organize in bi-distilled liquid water due to low-energy physical perturbations of various kinds. With the addition of infrared energy, these water nanostructures have also been shown capable of producing and conducting a significant flow of quasi-free electrons without the presence of exogenous ions [7].

The presence of a surplus of quasi-free electrons and the formation of coherent domains in liquid water-not predicted by standard views - may be described via quantum field theory [8, 9]. Our calculations above suggest modifications to the strictly quantum mechanical calculation of the Zeeman effect on a free electron state, thus obtaining a formal analogy with the Larmor precession formula. This result may explain the formation of coherent vortices in ensembles of free electrons in liquid water [10].

The most immediate possible extrapolation of our presented estimates would be to such large systems of free, or quasi-free, electrons. Such a realistic extrapolation of the results of our preliminary findings would require very strong theoretical and experimental efforts, which would be novel in their approach. These efforts have already begun and involve a group of dedicated colleagues, including the present authors.

[1] P. Kurian and C. Verzegnassi. Phys. Lett. A 380, 394-396 (2016).

[2] M. E. Peskin and D. V. Schroeder. An Introduction to Quantum Field Theory. Perseus, 1995.

[3] C. Verzegnassi. J. Mod. Phys. 6, 88-93 (2015).

[4] V. Kiran, S. R. Cohen, and R. Naaman. J. Chem. Phys. 146, 092302 (2017).

[5] D. Mishra, T. Z. Markus, R. Naaman, M. Kettner, B. Göhler, H. Zacharias, N. Friedman, M. Sheves, and C. Fontanesi. Proc. Natl. Acad. Sci. USA 110, 14872-14876 (2013).

[6] V. Elia, T.A. Yinnon, R. Oliva, E. Napoli, R. Germano, F. Bobba, and A. Amoresano. Water 8, 1-29 (2017).

[7] R. Germano, E. Del Giudice, A. De Ninno, V. Elia, C. Hison, E. Napoli, V. Tontodonato, F. P. Tuccinardi, and G. Vitiello. Key Engineering Materials 543, 455-459 (2013).

[8] E. Del Giudice and G. Vitiello. Phys. Rev. A 74, 022105 (2006).

[9] P. Kurian, A. Capolupo, T. J. A. Craddock, and G. Vitiello. arXiv:1608.08097 [physics.bio-ph] (2017).

[10] E. Del Giudice and G. Vitiello. Water 2, 133-141 (2011). 